\title{
Contact line arrest in solidifying spreading drops
}

\author{
Riëlle de Ruiter, ${ }^{1}$ Pierre Colinet, ${ }^{2}$ Philippe Brunet, ${ }^{3}$ Jacco H. Snoeijer, ${ }^{1,4}$ \\ and Hanneke Gelderblom ${ }^{1, *}$ \\ ${ }^{1}$ Physics of Fluids Group, Faculty of Science and Technology, MESA+ Institute for Nanotechnology, \\ University of Twente, P.O. Box 217, 7500 AE Enschede, The Netherlands \\ ${ }^{2}$ Transfers, Interfaces and Processes, Université Libre de Bruxelles, C.P. 165/67, Brussels, Belgium \\ ${ }^{3}$ Laboratoire Matière et Systèmes Complexes, Université Paris Diderot, Paris, France \\ ${ }^{4}$ Department of Applied Physics, Eindhoven University of Technology, \\ P.O. Box 513, 5600 MB Eindhoven, The Netherlands
}

(Received 18 November 2016; published 19 April 2017)

\begin{abstract}
When does a drop, deposited on a cold substrate, stop spreading? Despite the practical relevance of this question, for example, in airplane icing and three-dimensional metal printing, the detailed mechanism of arrest in solidifying spreading drops has remained debated. Here we consider the spreading and arrest of hexadecane drops of constant volume on two smooth wettable substrates: copper with a high thermal conductivity and glass with a low thermal conductivity. We record the spreading radius and contact angle in time for a range of substrate temperatures. The experiments are complemented by a detailed analysis of the temperature field near the rapidly moving contact line, by means of similarity solutions of the thermohydrodynamic problem. Our combined experimental and theoretical results provide strong evidence that the spreading of solidifying drops is arrested when the liquid at the contact line reaches a critical temperature, which is determined by the effect of kinetic undercooling.
\end{abstract}

DOI: 10.1103/PhysRevFluids.2.043602

\section{INTRODUCTION}

The spreading of liquid drops on a solid substrate that is below the liquid's freezing temperature is a phenomenon encountered in various applications. For example, raindrops that impinge and subsequently freeze on cold surfaces can negatively affect the operation of airplanes, wind turbines, or transmission lines. Anti-icing surfaces prevent this icing by minimizing the contact time between drop and substrate or delaying heterogeneous nucleation of the liquid [1-4]. Other applications can be found in the three-dimensional (3D) printing of micrometer-sized molten metal drops for precise fabrication [5,6], thermal spray coating [7], soldering [8], and lava flows [9].

The solidification of spreading drops is a challenging problem due to the combination of a moving contact line [10] with simultaneous heat transfer and phase transition [11]. More specifically, the problem is complicated by the singularity in both stress [12] and heat flux at the contact line [13-15]. Under isothermal conditions, the spreading of low-viscosity drops on a smooth complete wetting substrate exhibits two distinct regimes, in which the capillary-driven motion is resisted either by inertia or by viscous dissipation close to the contact line [16]. The spreading dynamics has been studied extensively, including the effects of partially wetting substrates [17,18], viscous drops [19], and complex substrates [20]. The bulk freezing of water drops deposited on a cold substrate has also attracted attention in recent years [21-25], with a focus on explaining the singular conical tip [22-24].

In this paper we focus on the short-time spreading dynamics of liquid drops on cold substrates. The moment of contact line arrest determines the final contact area of the drop with the substrate and its global shape. However, despite its importance in above-mentioned applications, the mechanism of solidifying spreading drops has remained subject to debate. The relevant question is the following:

\footnotetext{
*Corresponding author: h.gelderblom@utwente.nl
} 

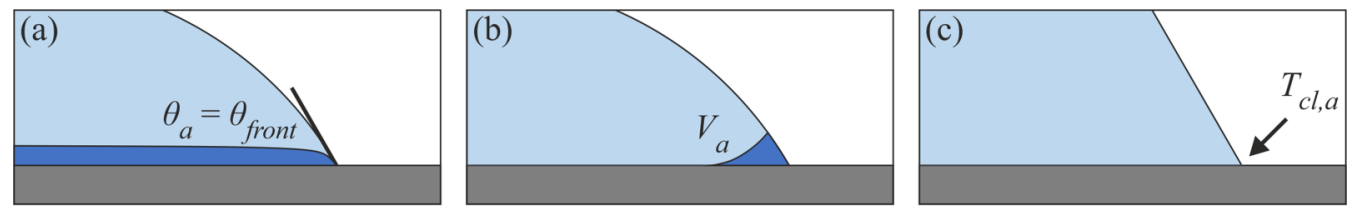

FIG. 1. Sketches of the different hypotheses for contact line arrest. Arrest occurs when (a) the contact angle of the spreading drop equals the angle of the freezing front at the contact line $[14,15]$, (b) a critical volume at the contact line is solidified [26], and (c) the liquid at the contact line reaches a critical temperature (present work).

When does a drop, deposited on a cold substrate, stop spreading? More strikingly, one may even wonder why the drop spreads at all, as one could expect immediate freezing at the initial contact of the drop with the undercooled substrate. A few interpretations can be found in the literature. Schiaffino and Sonin [14] studied the forced spreading of molten wax on a solid wax target. Microdrops were generated at a few kilohertz rate and impacted the substrate to form a spreading sessile liquid macrodrop. In their work it was assumed that a dynamic solidification front grows from the basal plane [see Fig. 1(a)]. The drop would then stop spreading as soon as the contact angle reaches the angle of the solidification front at the contact line. The experiments showed a dependence of the arrest contact angle on the Stefan number $\mathrm{St}=c \Delta T / L$ (the ratio of sensible heat to latent heat) as $\theta_{a} \propto \mathrm{St}^{1 / 2}$. Here $c$ is the specific heat capacity, $\Delta T$ is the difference between the melting temperature and the substrate temperature, and $L$ is the specific latent heat. However, a prediction of this angle is not possible due to the contact line singularity of the heat flux from the solidification front into the solid. A comparison to numerical calculations yielded a cutoff length scale of $0.1-1 \mu \mathrm{m}$ for their experimental conditions, but the physical interpretation of the cutoff remains questionable [15]. More recently, Tavakoli et al. [26] studied the forced spreading of alkanes on glass by dispensing the liquid at a rate of $5-600 \mu \mathrm{L} / \mathrm{min}$. In their work it was assumed that solidification starts from the contact line [see Fig. 1(b)], where the cooling time is taken to be the time after touchdown of the drop. The drop would then stop spreading once a critical volume of liquid at the contact line is solidified. For this criterion to be valid, the nucleus of the growing solidified volume needs to follow the moving contact line. It is not specified how this relates to the usual spreading, where fresh liquid is continuously supplied to the contact line. Both these mechanisms $[14,26]$ are based on solidification of a part of the drop as an arrest condition, so the latent heat of the liquid is an important parameter.

In this work we address the arrest condition by experiments of spreading hexadecane drops; the drops are gently deposited at constant volume on smooth wettable substrates of a different material. We report spreading curves and contact angle measurements of hexadecane drops spreading on substrates with high (copper) and low (glass) thermal conductivity (Sec. II). To explain our results, we propose a different mechanism, based on crystallization kinetics [see Fig. 1(c)]. A drop then is arrested when the liquid at the contact line reaches a certain critical temperature that depends on the velocity of the contact line (Sec. III). To account for the effect of the thermal properties of the substrate, we solve the Navier-Stokes and heat equations locally near the contact line and show how this changes the moment of arrest.

\section{EXPERIMENT}

\section{A. Method}

Figure 2(a) shows a sketch of the experimental setup. A liquid drop is formed at the end of the needle and gently deposited ( $\mathrm{We}=\rho_{l} v^{2} R_{0} / \sigma \ll 1$, with $\rho_{l}$ the density, $v$ the impact speed, $R_{0}$ the initial drop radius, and $\sigma$ the surface tension) onto the cold target substrate. We carried out spreading experiments with hexadecane $\left(\mathrm{C}_{16} \mathrm{H}_{34}\right.$, Sigma-Aldrich), a simple linear alkane with a well-defined freezing temperature $T_{f}=18^{\circ} \mathrm{C}$. The hexadecane is heated to an initial temperature $T_{i}=28^{\circ} \mathrm{C}$ 

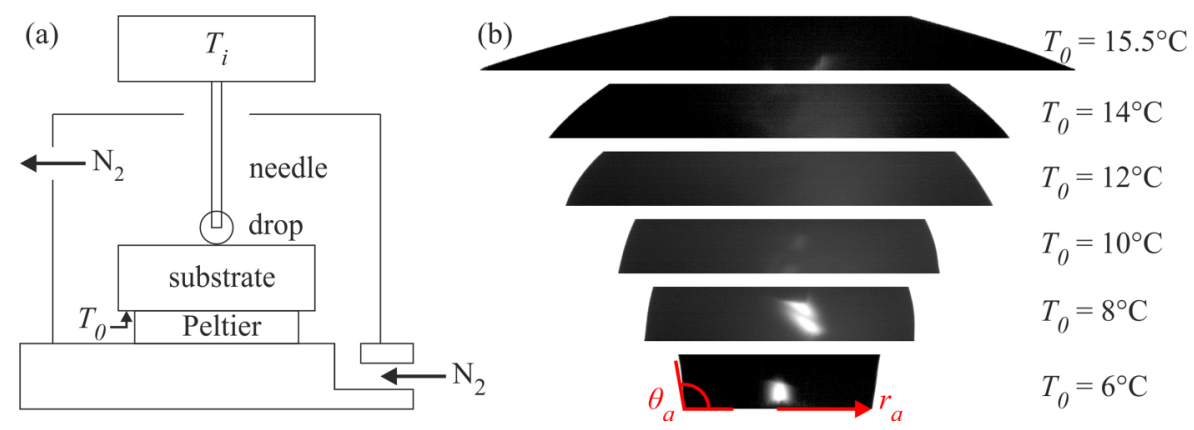

FIG. 2. (a) Sketch of the experimental setup (not to scale). (b) Snapshots at the moment of contact line arrest of (the lower part of) hexadecane drops spreading on a copper substrate for various substrate temperatures $T_{0}$. The arrest spreading radius $R_{a}$ and arrest contact angle $\theta_{a}$ are indicated in red.

before entering the needle [see Fig. 2(a)]. We verified that increasing the initial temperature up to $48^{\circ} \mathrm{C}$ does not have a significant influence on the spreading of the drops on a copper substrate. The temperature of the bottom of the substrate $T_{0}$ is controlled by a Peltier element and a thermistor. We ensure that the thickness of the substrates is larger than the characteristic thermal diffusion depth $\left(4 \alpha_{\mathrm{sub}} t\right)^{1 / 2}$ at the time scales of the experiment, such that the drop-substrate interface temperature $T_{\text {int }}$ remains constant. Here $\alpha_{\text {sub }}$ is the thermal diffusivity of the substrate. We performed the experiments on copper and soda-lime glass substrates. Condensation onto the cold substrate is largely prevented by using a dry nitrogen atmosphere. Substrates are thoroughly rinsed with ethanol before use. The copper substrate has such a high thermal conductivity $\left[k_{\text {sub }}=397.7 \mathrm{~W} /(\mathrm{m} \mathrm{K})\right]$ in comparison to hexadecane $\left[k_{l}=0.15 \mathrm{~W} /(\mathrm{m} \mathrm{K})\right]$ that it can be considered isothermal. Significant thermal gradients are much more likely in the glass substrate, as its thermal conductivity $\left[k_{\text {sub }}=1.0 \mathrm{~W} /(\mathrm{m} \mathrm{K})\right]$ is close to that of hexadecane. Table I gives an overview of the relevant properties for the liquid and solid hexadecane and the copper and glass substrates. For a discussion in terms of dimensionless parameters we refer the reader to Sec. III B.

A drop of fixed size (for copper $R_{0}=725 \pm 8 \mu \mathrm{m}$ and for glass $R_{0}=834 \pm 17 \mu \mathrm{m}$ ) is formed at the tip of a needle and lowered onto the target substrate. Bond numbers Bo $=\rho g R_{0}^{2} / \sigma$, with $g$ the gravitational acceleration, are smaller than unity such that we can neglect the effect of gravity. Spreading and arrest of the drops is visualized with a high-speed camera (Photron SA-7 or Photron SA-1) recording at 10-20 kiloframes/s and a Navitar $12 \times$ zoom lens. We use a custom MATLAB routine that extracts the drop contours via thresholding. The contours are analyzed to extract the spreading radius and the contact angle is determined by a linear fit to the contour at the contact line, typically resolved on a scale of $30 \mu \mathrm{m}$ from the contact line.

TABLE I. Properties of the liquid, solid, and substrate.

\begin{tabular}{lcccc}
\hline \hline & \multirow{2}{*}{$\begin{array}{c}\text { Liquid }(i=l) \\
\text { Property }\end{array}$} & $\begin{array}{c}\text { Solid }(i=s) \\
\text { Hexadecane }\end{array}$ & Hexadecane & Substrate $(i=$ sub $)$ \\
\cline { 5 - 6 } viscosity $\mu(\mathrm{Pas})$ & 0.003 & & Soda-lime glass \\
surface tension $\sigma(\mathrm{N} / \mathrm{m})$ & 0.028 & & & \\
density $\rho_{i}\left(\mathrm{~kg} / \mathrm{m}^{3}\right)$ & 774 & 833 & 8960 & 2479 \\
specific heat capacity $c_{i}[\mathrm{~J} /(\mathrm{kg} \mathrm{K})]$ & 2310 & 1800 & 386 & 760 \\
thermal diffusivity $\alpha_{i}\left(\mathrm{~m}^{2} / \mathrm{s}\right)$ & $8.4 \times 10^{-8}$ & $1.0 \times 10^{-7}$ & $1.2 \times 10^{-4}$ & $5.3 \times 10^{-7}$ \\
thermal conductivity $k_{i}[\mathrm{~W} /(\mathrm{m} \mathrm{K})]$ & 0.15 & 0.15 & 397.7 & 1.0 \\
latent heat $L(\mathrm{~J} / \mathrm{kg})$ & \multicolumn{2}{c}{$2.3 \times 10^{5}$} & & \\
freezing temperature $T_{f}\left({ }^{\circ} \mathrm{C}\right)$ & \multicolumn{2}{c}{18} & \\
\hline \hline
\end{tabular}



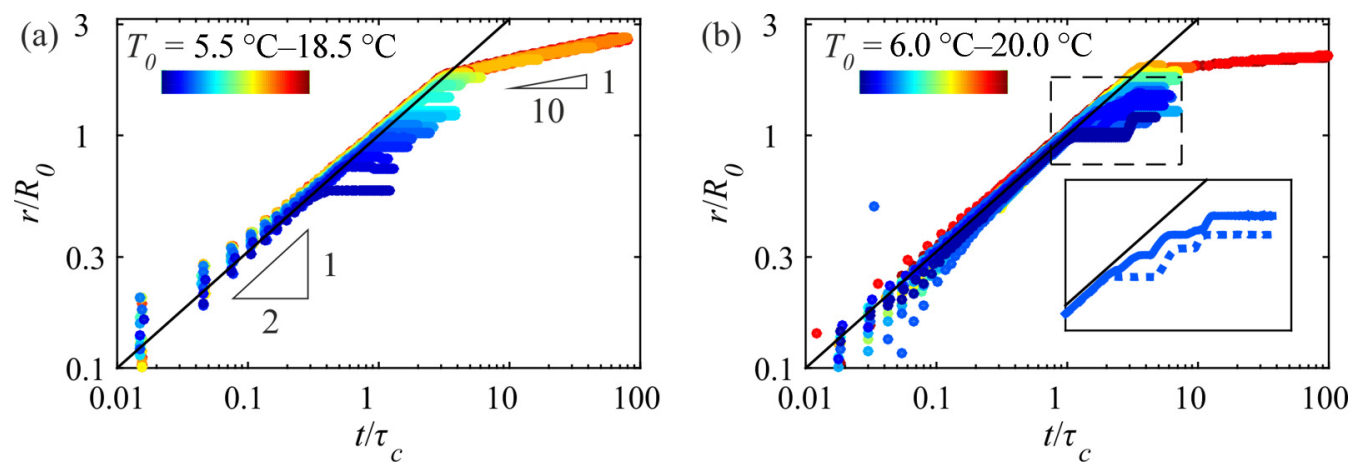

FIG. 3. Spreading radius versus time for a range of substrate temperatures (a) on a copper substrate and (b) on a glass substrate $\left(T_{i}=28^{\circ} \mathrm{C}\right)$. The inset in (b) shows a close-up of the stick-slip behavior on glass for $T_{0}=8^{\circ} \mathrm{C}$ (left and right contact lines). The black line is the spreading law in the inertiocapillary regime [Eq. (1)].

\section{B. Results}

Whereas drops on a warm substrate of $T_{0}>T_{f}$ spread into a very thin layer, drops on a colder substrate get quickly arrested on time scales of the order of a few milliseconds. Snapshots of the drops at the moment of contact line arrest are shown in Fig. 2(b) for different substrate temperatures $T_{0}$. For a colder substrate, spreading stops earlier and the footprint radius of the drop at arrest is smaller. After the arrest shown in Fig. 2(b), there is still some dynamics. Namely, the bulk of the drop is still coming down due to inertia. The contact angle then increases, while the footprint area of the drop remains constant. After the entire drop is solidified, hexadecane drops show a dimple, a small depression at the top of the drop, due to the increase in density upon solidification. This is in contrast to water drops that form a conical tip due to a decrease in density [22-24].

It is important to note that bulk solidification of the drop occurs at much larger time scales than contact line arrest. The Stefan numbers of the liquid and solid (ice) phases are both small, $\mathrm{St}_{l}=c_{l}\left(T_{i}-T_{f}\right) / L<1$ and $\mathrm{St}_{s}=c_{s}\left(T_{f}-T_{0}\right) / L<1$, with $c_{l, s}$ the specific heat capacity of the liquid and solid phases and $L$ the specific latent. The freezing time of the global drop is thus dominated by the removal of latent heat and can be estimated by dividing the latent heat $\rho V L$ by the approximate heat flux $\kappa_{s}\left(T_{f}-T_{0}\right) / R_{0}$, resulting in $t_{f} \propto R_{0}^{2} / \alpha_{s} \mathrm{St}_{s}$. Here it is assumed that heat transfer through the ice phase is slow compared to heat transfer through the substrate. Bulk freezing times are of the order of a minute, which shows that there is a large separation of time scales between bulk freezing and contact line arrest.

The spreading dynamics for hexadecane drops on copper are reported in Fig. 3(a). The spreading radius $r$ is rescaled with the initial drop radius $R_{0}$ and the time after contact $t$ is rescaled with the capillary time scale $\tau_{c}=\left(\rho_{l} R_{0}^{3} / \sigma\right)^{1 / 2}$, which is 3-4 ms in our case. For substrate temperatures above the freezing temperature (see the red data points), we recover at short time scales the isothermal spreading law of the inertiocapillary regime [16-20]

$$
r / R_{0} \propto\left(t / \tau_{c}\right)^{1 / 2}
$$

At later stages the spreading dynamics is ruled by a balance between capillarity and viscous stress. This is the Tanner regime [16] with a spreading exponent of $1 / 10$. For cold substrates, we observe that the spreading radius initially closely follows the isothermal law(s), up to the moment when the contact line suddenly stops. The colder the substrate, the earlier arrest occurs. For substrate temperatures $T_{0} \leqslant 5^{\circ} \mathrm{C}$, drops form a solidified layer before touching the substrate and spreading is not observed.

The spreading curves for hexadecane drops on glass are reported in Fig. 3(b). For glass substrate temperatures $T_{0} \leqslant 12^{\circ} \mathrm{C}$, the drops exhibit stick-slip behavior (see the inset). The contact line is 

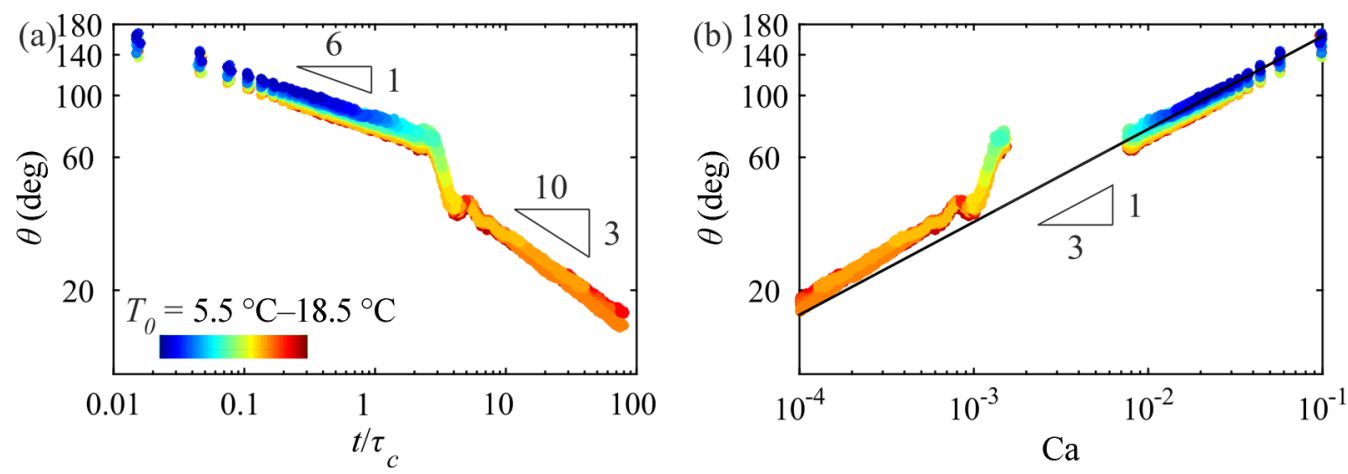

FIG. 4. Contact angle versus (a) time or (b) capillary number for a range of substrate temperatures on a copper substrate $\left(T_{i}=28^{\circ} \mathrm{C}\right)$. The contact angle is plotted up to the moment of contact line arrest. The black line in (b) is the Cox-Voinov law [Eq. (3)] as fitted in the inertiocapillary regime with $\ln \left(R_{0} / l\right)=25.7$.

pinned at either side, not necessarily simultaneously, after which the contact angle increases until depinning occurs. The drop goes through a series of pinning and depinning events before the final spreading radius is reached. In the interpretation of the data, we will focus on the moment of initial pinning, i.e., when the arrest condition is attained for the first time.

We now turn to the time evolution of the contact angle. For substrates of $T_{0}>T_{f}$, the contact angle decreases during spreading from $180^{\circ}$ to $0^{\circ}$ on a completely wetting substrate. Our field of view captures this dynamics down to a contact angle of $20^{\circ}$ [see the red data in Fig. 4(a)]. A further decrease of the contact angle occurs outside the field of view. Again we observe two different scaling regimes corresponding to inertial (large $\theta$ ) and viscous (small $\theta$ ) spreading, respectively. For cold substrates, the contact angle follows the same scaling, until the contact line stops.

In Fig. 4(b) the contact angle data are plotted versus the capillary number $\mathrm{Ca}=\mu v_{c l} / \sigma$. Here $\mu$ is the viscosity and $v_{c l}$ is the velocity of the contact line as derived from the spreading law [Eq. (1)]. We recover the classical Cox-Voinov behavior [27,28]

$$
\theta^{3}=9 \mathrm{Ca} \ln \left(R_{0} / l\right)
$$

in both regimes, as also shown by Schiaffino and Sonin for solidifying drops [14]. The deviations around $70^{\circ}-40^{\circ}$ are caused by the transition between both regimes and the capillary wave arriving at the contact line. The logarithmic factor $\ln \left(R_{0} / l\right)$, with $l$ the characteristic microscopic length scale at which molecular processes regularize the viscous singularity, is determined from the curves in Fig. 4(b) to be 25.7. Similarly large values have been measured for water drops and could point to additional dissipative mechanisms (beyond hydrodynamics) near the contact line [29,30]. The scaling in Fig. 4(a) $\left(\theta \propto t^{-1 / 6}\right.$ and $\left.\theta \propto t^{-3 / 10}\right)$ is a direct consequence of combining the Cox-Voinov behavior $\left(\theta \propto \mathrm{Ca}^{1 / 3}\right)$ with the spreading laws in the inertiocapillary $\left(r \propto t^{1 / 2}\right)$ and viscocapillary $\left(r \propto t^{1 / 10}\right)$ regimes.

Remarkably, as shown by both the spreading radius and the contact angle data, the drop dynamics prior to arrest is experimentally indistinguishable from isothermal spreading without solidification. We therefore do not expect a dramatic increase of the viscosity upon cooling down to the freezing temperature, which is consistent with previous results for other alkanes [31].

Based on our data, we now quantify the conditions of arrest. The arrest spreading radius can be very precisely determined from Fig. 3. The value of the contact angle increases sharply after contact line arrest. We therefore define the minimum contact angle before arrest as the arrest contact angle. In Fig. 5 we show the spreading radius $r_{a}$ and contact angle $\theta_{a}$ at contact line arrest as a function of the substrate undercooling $\Delta T=T_{f}-T_{0}$. For a deeper substrate undercooling, contact line arrest occurs faster, which results in a decrease of the arrest spreading radius and an increase of the arrest contact angle. 

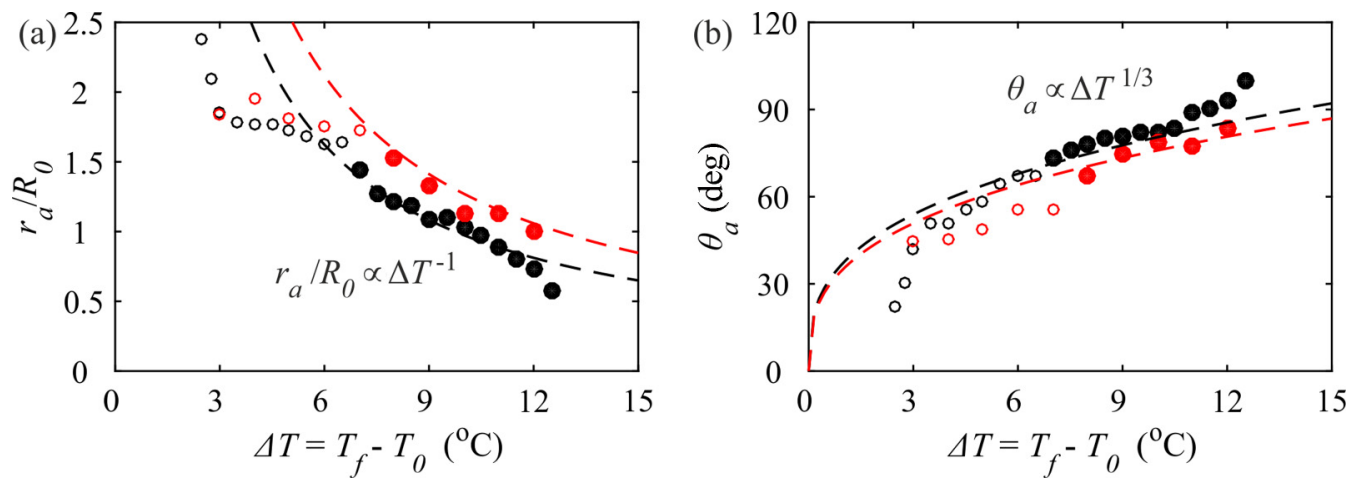

FIG. 5. (a) Arrest spreading radius and (b) arrest contact angle versus the substrate undercooling $\Delta T=$ $T_{f}-T_{0}$. Black indicates the copper substrate (properties at arrest) and red the glass substrate (properties at first pinning event). Closed symbols indicate drops that get arrested in the inertiocapillary regime, while open symbols indicate drops that get arrested in the viscocapillary regime or during the transition between both regimes. Dashed lines show the model with kinetic undercooling (see Sec. III). A kinetic coefficient of $\kappa=0.011 \mathrm{~m} /(\mathrm{s} \mathrm{K})$ is used.

We compare the arrest properties of hexadecane drops spreading on copper (black) and on glass (red). In the latter case, thermal gradients are present inside the substrate due to the lower thermal conductivity and the temperature on top of the substrate is expected to deviate significantly from the temperature applied to the bottom of the substrate. On glass, the cooling of the drops is therefore less efficient, resulting in a small shift to larger arrest spreading radius and smaller arrest contact angle for the same substrate temperature.

\section{INTERPRETATION}

An important observation is that the motion of the contact line is completely unaffected by the cooling prior to the arrest. This implies that the spreading has the usual hydrodynamic features, including a rapid motion that is due to a continuous supply of fresh liquid near the contact line. Based on these observations, we propose a mechanism that is consistent with the kinematics of such rapid contact line motion and therefore differs, e.g., from the criteria proposed by Schiaffino and Sonin [14] and Tavakoli et al. [26]. In particular, we assume that the drop stops spreading when the liquid at the contact line reaches a certain critical temperature for arrest $T_{c l, a}$, which differs from the bulk freezing temperature. This change is attributed to the effect of kinetic undercooling, which results in a lower solidification temperature. A secondary effect is the substrate conductivity (copper versus glass), which is considered in a thermohydrodynamic model.

\section{A. Spreading on a copper substrate}

A copper substrate has such a high thermal conductivity that it can be considered isothermal during the drop spreading. By continuity, i.e., neglecting any thermal resistance between liquid and substrate, the liquid at the contact line is thus also at $T_{c l}=T_{0}$. We first assume that the critical temperature for arrest $T_{c l, a}$ would be equal to the bulk freezing temperature of the liquid $T_{f}$. Every drop that approaches a copper substrate with $\Delta T>0\left(T_{0}<T_{f}\right)$ would then be predicted to freeze instantaneously with $r_{a}=0$ and $\theta_{a}=180^{\circ}$, due to the large thermal conductivity of copper. However, the experimental arrest contact angles turn out to be significantly smaller [see Figs. 2(b) and 5(b)]. We thus need to take into account an effect that delays the contact line arrest.

Our main hypothesis in this work is that the dominant effect is kinetic undercooling due to the fast contact line motion during the initial spreading. Crystallization of the liquid becomes the rate-limiting step in case of nonequilibrium solidification. A velocity of the freezing front can in fact 
only be sustained as long as molecules attach faster to the front than they detach, which requires undercooling of the liquid. For small kinetic undercooling, the undercooling is linearly dependent on the velocity of the freezing front: $T_{f}-T_{\text {front }}=v_{\text {front }} / \kappa[32-34]$. Here $\kappa$ is the kinetic coefficient, which is a property of the liquid. A smaller kinetic coefficient (mobility) means that it is more difficult for molecules to attach to the growing crystal and a larger undercooling is thus needed in order to freeze the liquid. We assume here that the contact line of our spreading drops can only be arrested if the freezing front velocity becomes equal to the velocity of the contact line so that $v_{\text {front }}=v_{c l, a}$ (otherwise, any crystal nucleus would be left behind the moving contact line). A significant substrate undercooling is thus necessary during the first stages of drop spreading, due to the very fast motion of the contact line at that moment. With this assumption, the arrest condition can thus be written

$$
T_{c l}=T_{c l, a}=T_{f}-v_{c l, a} / \kappa .
$$

The contact line slows down during drop spreading, resulting in an increase of $T_{c l, a}$. We now obtain expressions for the arrest radius and the arrest contact angle at the moment the arrest condition is met. The relationship between the rescaled spreading radius and the contact line velocity can be derived from the spreading law in the inertiocapillary regime [Eq. (1)]. As the prefactor is determined to be 1.0 from a fit to the data in Fig. 3, this yields $v_{c l}=\left(R_{0} / 2 \tau_{c}\right) /\left(r / R_{0}\right)$. Combining this with Eq. (3) and $T_{c l}=T_{0}$ (see above), we obtain for the arrest spreading radius

$$
r_{a} / R_{0}=R_{0} / 2 \tau_{c} \kappa \Delta T \propto \Delta T^{-1} .
$$

Combining the Cox-Voinov law [Eq. (2)] with the arrest criterion [Eq. (3)] and $T_{c l}=T_{0}$, we obtain for the arrest contact angle

$$
\theta_{a}=\left[9 \mu \ln \left(R_{0} / l\right) \kappa \Delta T / \sigma\right]^{1 / 3} \propto \Delta T^{1 / 3} .
$$

These expressions are shown as the dashed black lines in Figs. 5(a) and 5(b), respectively. It should be noted that Eq. (4) is only valid for the inertiocapillary regime (closed symbols). A similar relation can be found for the viscocapillary regime $\left(r_{a} / R_{0} \propto \Delta T^{-1 / 9}\right)$. However, since most open symbols are actually acquired during the transition between both regimes, we limit the fitting of $\kappa$ to the inertiocapillary regime. On the other hand, Eq. (5) should be valid in both regimes. Our model gives good agreement with the data for $\kappa=0.011 \mathrm{~m} /(\mathrm{s} \mathrm{K})$. This fitting parameter is determined from the data for the arrest spreading radius, as its experimental determination is more accurate than that of the arrest contact angle. We have not been able to find experimental data for $\kappa$ of hexadecane in the literature.

\section{B. Spreading on a glass substrate}

\section{Corner flow model with heat transfer}

As shown in Fig. 5, drops spreading on the glass substrate get arrested later, at larger arrest spreading radius and smaller arrest contact angle. The substrate temperature $T_{0}$ is controlled at the bottom of the substrate. The temperature of the liquid at the contact line is somewhat above the substrate temperature $T_{c l}>T_{0}$ due to the thermal gradients across the glass substrate. Therefore, in this case the heat transfer from the liquid to the substrate, including convective heat transfer within the liquid, needs to be considered to determine the arrest conditions. To this end, we solve the Navier-Stokes equations (in the liquid) and heat equations (in the substrate and the liquid) in a two-dimensional wedge geometry locally near the moving contact line [see Fig. 6(a)]. We consider a wedge of liquid with contact angle $\theta$ moving with contact line velocity $v_{c l}$ over a substrate of which the temperature $T_{0}$ is controlled at the bottom, while the initial drop temperature $T_{i}$ prevails far from the contact line in the liquid.

More precisely, we will take advantage of a remarkable coincidence: In the inertiocapillary regime, the characteristic length scales of the hydrodynamics (convection) and the thermal diffusion (conduction) both behave as $t^{1 / 2}$. Despite the highly unsteady nature of the process, the relative strength of convection and diffusion therefore remains constant over time and is governed by material parameters that will be identified below. For now, we proceed by introducing the hydrodynamic length 

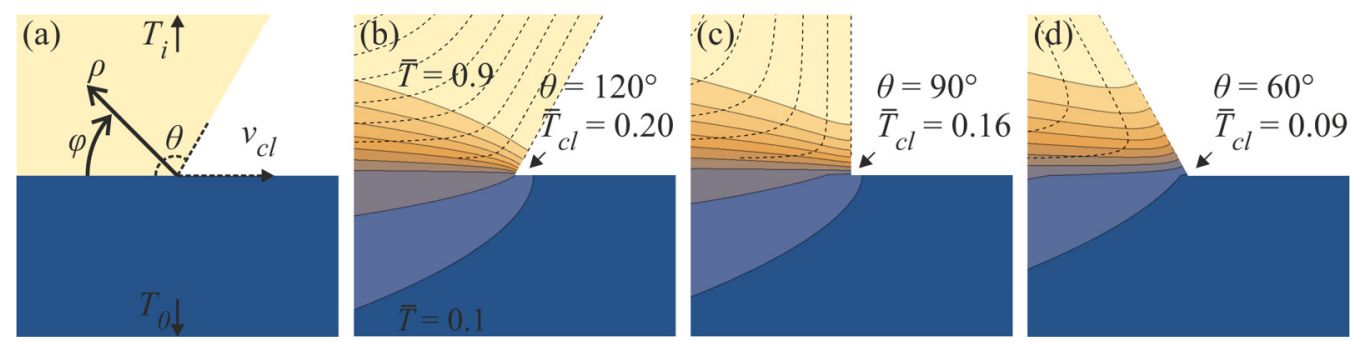

FIG. 6. (a) Geometry in the contact line region for calculation of the temperature field. (b)-(d) Isotherms (solid lines) and streamline patterns (dashed lines) for hexadecane drops on a glass substrate with a contact angle of (b) $\theta=120^{\circ}$, (c) $\theta=90^{\circ}$, and (d) $\theta=60^{\circ}$. The rescaled contact line temperatures are indicated.

$l_{v}=(v t)^{1 / 2}$, where $v$ is the liquid kinematic viscosity, and investigate the dynamics on this scale near the contact line. Note that a local formulation that includes only the contact line region requires $l_{v} \ll r$, which is equivalent to the condition $\sqrt{\mathrm{Oh}} \ll 1$, where $\mathrm{Oh}=v \tau_{c} / R_{0}^{2}=\mu /\left(\rho_{l} \sigma R_{0}\right)^{1 / 2}$ is the Ohnesorge number. We also neglect the influence of the contact angle decrease compared to the contact line velocity at the scale considered, i.e., $l_{v}|\dot{\theta}| \ll v_{c l}=\dot{r}$. Using Eqs. (1) and (2), this can actually be recast into the form $\sqrt{\mathrm{Oh}} \ll 3 / \theta$, which is also satisfied at small Ohnesorge number (as the maximal value of $\theta$ is $\pi$ ).

Given the absence of any geometrical length scale locally near the contact line, a self-similar (penetrationlike) solution of the complete thermohydrodynamic problem may be obtained by scaling lengths by $l_{v}=(v t)^{1 / 2}$ and velocities by $l_{v} / t=(v / t)^{1 / 2}$, noting that the dimensionless contact line speed $\hat{v}_{c l}=v_{c l} t / l_{v}=1 / 2 \sqrt{\mathrm{Oh}}$ is then a constant in the inertiocapillary regime of interest. The temperatures are offset by $T_{0}$ and scaled by the temperature difference $T_{i}-T_{0}$. Thus, we represent velocity and temperature fields in the moving frame as

$$
\begin{aligned}
\vec{V}^{*} & =\sqrt{v / t}\left[\hat{v}_{c l} \overrightarrow{1}_{x}+\vec{V}(\rho, \varphi)\right], \\
T_{l, \text { sub }}^{*} & =T_{0}+\left(T_{i}-T_{0}\right) T_{l, \text { sub }}(\rho, \varphi),
\end{aligned}
$$

where asterisks mark dimensional quantities, $(\rho, \varphi)$ are the dimensionless polar coordinates near the contact line [see Fig. 6(a)], $\overrightarrow{1}_{x}$ is the unit vector along its direction of motion, and subscripts $l$ and $s u b$ denote liquid and substrate, respectively. Substituting Eqs. (6) and (7) into continuity, Navier-Stokes, and energy equations then yields the system

$$
\begin{gathered}
\vec{\nabla} \cdot \vec{V}=0 \\
-\frac{\vec{V}}{2}-\frac{\rho}{2} \frac{\partial \vec{V}}{\partial \rho}+(\vec{V} \cdot \vec{\nabla}) \vec{V}=\Delta \vec{V}-\vec{\nabla} p \\
-\frac{\rho}{2} \frac{\partial T_{l}}{\partial \rho}+(\vec{V} \cdot \vec{\nabla}) T_{l}=\operatorname{Pr}^{-1} \Delta T_{l} \\
-\frac{\rho}{2} \frac{\partial T_{\text {sub }}}{\partial \rho}+\hat{v}_{c l}\left(\cos \varphi \frac{\partial T_{\text {sub }}}{\partial \rho}-\frac{\sin \varphi}{\rho} \frac{\partial T_{\text {sub }}}{\partial \varphi}\right)=\alpha \operatorname{Pr}^{-1} \Delta T_{\text {sub }}
\end{gathered}
$$

where $p$ is the dimensionless pressure (redefined to incorporate a deceleration term), $\alpha=\alpha_{\text {sub }} / \alpha_{l}$ is the ratio of thermal diffusivities, and $\operatorname{Pr}=v / \alpha_{l}$ is the Prandtl number of the liquid.

Now boundary conditions at the substrate-liquid interface $\varphi=0$, at the liquid-air interface $\varphi=\theta$, and at the substrate-air interface $\varphi=-\pi$ are (denoting by $U$ and $V$ the components of $\vec{V}$ along $\rho$ 
and $\varphi$, respectively)

$$
\begin{gathered}
U=\hat{v}_{c l}=1 / 2 \sqrt{\mathrm{Oh}}, \quad V=0, \quad T_{\text {sub }}=T_{l}, \quad k \partial T_{\text {sub }} / \partial \varphi=\partial T_{l} / \partial \varphi \quad \text { for } \varphi=0, \\
V=0, \quad \rho^{2} \partial\left(\rho^{-1} V\right) / \partial \rho+\partial U / \partial \varphi=0, \quad \partial T_{l} / \partial \varphi=0 \quad \text { for } \varphi=\theta \\
\partial T_{\text {sub }} / \partial \varphi=0 \quad \text { for } \varphi=-\pi,
\end{gathered}
$$

where $k=k_{\text {sub }} / k_{l}$ is the substrate-to-liquid ratio of thermal conductivities and it has been assumed that heat transfer to the ambient is negligible, implying that the isotherms within the drop and the substrate are always perpendicular to their interface with air. Note that it is in the first of Eqs. (13) that the velocity induced by the closing of the wedge has been neglected, as discussed above, while the second of Eqs. (13) expresses that the liquid-air interface is stress-free. Finally, boundary/initial conditions are needed in the far field $\rho \rightarrow \infty$, where we impose $T_{\text {sub }} \rightarrow 0, T_{l} \rightarrow 1$, and $\vec{V} \rightarrow 0$.

The problem (8)-(14) is thus fully defined by $\theta$, the liquid parameters $\mathrm{Oh}=0.023$ (a value deemed to be small enough and for which $\hat{v}_{c l}=3.3$ ) and $\operatorname{Pr}=46$, and the liquid-substrate interaction parameters $\alpha=6.3$ and $k=6.6$. These values are for hexadecane drops on a soda-lime glass substrate. For a copper substrate, $\alpha=1370$ and $\kappa=2640$ and we assume that the substrate is isothermal. This can also be seen by comparing the convection in the liquid with the conduction in the solid; the relevant dimensionless parameter is $r / l_{\alpha}$, which compares the spreading radius (convection) to the thermal diffusion length in the solid (conduction). This yields $r / l_{\alpha}=(\operatorname{Pr} /[\alpha \mathrm{Oh}])^{1 / 2}$, which is 1.2 for copper and 17.8 for glass.

The physical properties are taken to be independent of temperature. We solve this boundaryvalue problem numerically, using a streamfunction/vorticity formulation and finite differences [with nonequidistant mesh to handle the fast variations occurring near the contact line $\rho \rightarrow 0$, where we also use classical corner solutions (see, e.g., [12]) in order to reach better convergence].

\section{Mechanism}

For every possible arrest contact angle $\theta_{a}$ we are now interested in the temperature of the liquid at the contact line. Figures 6(b)-6(d) give three typical examples for $\theta_{a}=120^{\circ}, 90^{\circ}$, and $60^{\circ}$. For small contact angles $\theta \rightarrow 0^{\circ}$, the rescaled contact line temperature $\bar{T}_{c l}=\left(T_{c l}-T_{0}\right) /\left(T_{i}-T_{0}\right)$ is close to zero, thus $T_{c l} \approx T_{0}$. In those cases there is not much fluid above the contact line that needs to be cooled down and the results are close to the results for a substrate with large thermal conductivity. If the wedge contact angle increases, $T_{c l}$ increases. Note that the coldest point in the liquid is always found at the contact line.

We assume that the spreading of a drop can be considered as a sequence of quasisteady self-similar solutions of Eqs. (8)-(14), with decreasing contact angle. Thus, the temperature of the liquid at the contact line is expected to decrease during spreading. Simultaneously, the kinetic undercooling reduces during spreading as the contact line slows down, increasing the critical temperature for arrest (as on the copper substrate). For some critical contact angle, the condition for contact line arrest (3) is met and the contact line stops. The results are shown as the red dashed lines in Fig. 5. Note that these numerical results are only valid in the inertiocapillary regime, as $r \propto t^{1 / 2}$ is implemented. For the lowly thermal conductive glass substrate, we used the same value for the kinetic coefficient as for the highly thermal conductive copper substrate, $\kappa=0.011 \mathrm{~m} /(\mathrm{s} \mathrm{K})$, as it is a property of the liquid only. Hence, accounting for the increase in $T_{c l}$ due to convection or diffusion compared to $T_{0}$, we indeed find a shift towards larger spreading radii and smaller contact angles on glass, in agreement with the experiments. The numerical results are rather robust against small variations in the initial drop temperature. A decrease from $T_{i}=28{ }^{\circ} \mathrm{C}$ to $T_{i}=23{ }^{\circ} \mathrm{C}$ causes the curve for the arrest radius to shift downward about the symbol size. 


\section{CONCLUSION AND DISCUSSION}

We studied the spreading of hexadecane drops on cold copper and glass substrates by measuring the spreading radius and contact angle evolution in time for a range of substrate temperatures. Drops are arrested more quickly for lower substrate temperatures and for a higher substrate thermal conductivity. We put forward a mechanism based on the kinetics of crystallization to rationalize our measurements of the moment of initial contact line arrest. We have shown that our experimental data are well described by considering a critical temperature of the liquid at the contact line and including kinetic undercooling, i.e., a nonequilibrium solidification temperature lower than the bulk freezing temperature.

For many practical applications, it would be interesting to investigate whether this model can be extended to impacting drops with a nonzero Weber number [8,35-38]. In many cases this involves the impact of a material of high thermal conductivity (i.e., the 3D printing of metals) onto a material of lower thermal conductivity such that thermal gradients in the substrate should again be considered with a convection-diffusion model. During impact, the early time spreading radius also increases with the square root of time, but now with the impact time $\tau_{i}=2 R_{0} / v$ as the characteristic time scale. In the simplest case, we expect that the contact line velocity again yields an estimation of the required undercooling of the liquid at the contact line to obtain arrest. The validation of this model for nonzero Weber numbers remains a challenge for future study.

Literature values for the kinetic coefficient are sparse. Experimental determination [39] is difficult, due to the necessity of simultaneously tracking the movement of the interface and to measure very small temperature variations. Most values reported are obtained from molecular dynamics simulations [40-42], where, for example, the undercooling is maintained by the use of thermostats and the velocity of the interface is computed by monitoring the total potential energy of the system [43]. The simulations are mainly limited to metals, for which values of $0.1-1 \mathrm{~m} /(\mathrm{s} \mathrm{K})$ are found. The order of magnitude of these values is not too different from the fitted value for hexadecane, $\kappa=0.011 \mathrm{~m} /(\mathrm{s} \mathrm{K})$. Values for polymers are not available, but we expect them to be lower due to an increase in molecular size, hence slower attachment kinetics.

To close, let us briefly reflect on the various proposed mechanisms for contact line arrest during solidification. In the present work we have attempted to incorporate the rapid wetting motion in the arrest condition, by means of a kinetic coefficient. Doing so, we have omitted the effect of latent heat. This can be rationalized since the volume considered near the contact line is very small such that the latent heat is expected not to be the limiting factor for solidification. This point of view is significantly different from the mechanism proposed by Tavakoli et al. [26], which is based on the latent heat produced by a critical volume near the contact line. A key assumption of that theory is that this volume starts to grow at the moment of touchdown of the drop and that it moves along with at the contact line. However, since prior to arrest we observe no difference with respect to usual spreading, where liquid is continuously transported towards the contact line, the kinetics of this mechanism needs to be clarified. Direct measurement of the kinetic coefficient, or of the geometry of the freezing fronts, is necessary to decisively resolve the challenging problem of contact line arrest.

\section{ACKNOWLEDGMENTS}

This work is part of an Industrial Partnership Programme of the Foundation for Fundamental Research on Matter, which is financially supported by the Netherlands Organisation for Scientific Research. This research program is cofinanced by ASML. P.C. acknowledges financial support from the Fonds de la Recherche Scientifique - F.N.R.S. and from BELSPO through IAP 7/38 MicroMAST Network.

[1] P. Tourkine, M. Le Merrer, and D. Quéré, Delayed freezing on water repellent materials, Langmuir 25, 7214 (2009). 
[2] L. Mishchenko, B. Hatton, V. Bahadur, J. A. Taylor, T. Krupenkin, and J. Aizenberg, Design of ice-free nanostructured surfaces based on repulsion of impacting water droplets, ACS Nano 4, 7699 (2010).

[3] J. C. Bird, R. Dhiman, H. M. Kwon, and K. K. Varanasi, Reducing the contact time of a bouncing drop, Nature (London) 503, 385 (2013).

[4] M. J. Kreder, J. Alvarenga, P. Kim, and J. Aizenberg, Design of anti-icing surfaces: Smooth, textured or slippery?, Nat. Rev. Mater. 1, 15003 (2016).

[5] M. Vaezi, H. Seitz, and S. F. Yang, A review on 3D micro-additive manufacturing technologies, Int. J. Adv. Manu. Technol. 67, 1721 (2013).

[6] C. W. Visser, R. Pohl, C. Sun, G. W. Roemer, B. H. in 't Veld, and D. Lohse, Toward 3D printing of pure metals by laser-induced forward transfer, Adv. Mater. 27, 4087 (2015).

[7] P. Fauchais, M. Fukumoto, A. Vardelle, and M. Vardelle, Knowledge concerning splat formation: An invited review, J. Thermal Spray Technol. 13, 337 (2004).

[8] D. Attinger, Z. Zhao, and D. Poulikakos, An experimental study of molten microdroplet surfacce deposition and solidification: Transient behavior and wetting angle dynamics, J. Heat Transfer 122, 544 (2000).

[9] R. W. Griffiths, The dynamics of lava flows, Annu. Rev. Fluid Mech. 32, 477 (2000).

[10] J. H. Snoeijer and B. Andreotti, in Moving Contact Lines: Scales, Regimes, and Dynamical Transitions, edited by S. H. Davis and P. Moin, special issue of Annu. Rev. Fluid Mech. 45, 269 (2013).

[11] H. S. Carslaw and J. C. Jaeger, Conduction of Heat in Solids, 2nd ed. (Oxford University Press, New York, 1959).

[12] C. Huh and L. E. Scriven, Hydrodynamic model of steady movement of a solid/liquid/fluid contact line, J. Colloid Interface Sci. 35, 85 (1971).

[13] D. M. Anderson and S. H. Davis, Local fluid and heat flow near contact lines, J. Fluid Mech. 268, 231 (1994).

[14] S. Schiaffino and A. A. Sonin, Motion and arrest of a molten contact line on a cold surface: An experimental study, Phys. Fluids 9, 2217 (1997).

[15] S. Schiaffino and A. A. Sonin, On the theory for arrest of an advancing molten contact line on a cold solid of the same material, Phys. Fluids 9, 2227 (1997).

[16] A. L. Biance, C. Clanet, and D. Quere, First steps in the spreading of a liquid drop, Phys. Rev. E 69, 016301 (2004).

[17] K. G. Winkels, J. H. Weijs, A. Eddi, and J. H. Snoeijer, Initial spreading of low-viscosity drops on partially wetting surfaces, Phys. Rev. E 85, 055301 (2012).

[18] J. C. Bird, S. Mandre, and H. A. Stone, Short-Time Dynamics of Partial Wetting, Phys. Rev. Lett. 100, 234501 (2008).

[19] A. Eddi, K. G. Winkels, and J. H. Snoeijer, Short time dynamics of viscous drop spreading, Phys. Fluids 25, 013102 (2013).

[20] B. B. J. Stapelbroek, H. P. Jansen, E. S. Kooij, J. H. Snoeijer, and A. Eddi, Universal spreading of water drops on complex surfaces, Soft Matter 10, 2641 (2014).

[21] D. M. Anderson, M. G. Worster, and S. H. Davis, The case for a dynamic contact angle in containerless solidification, J. Cryst. Growth 163, 329 (1996).

[22] W. W. Schultz, M. G. Worster, and D. M. Anderson, in Interactive Dynamics of Convection and Solidification, edited by P. Ehrhard, D. S. Riley, and P. H. Steen (Springer, Dordrecht, 2001).

[23] J. H. Snoeijer and P. Brunet, Pointy ice-drops: How water freezes into a singular shape, Am. J. Phys. 80, 764 (2012).

[24] A. G. Marin, O. R. Enriquez, P. Brunet, P. Colinet, and J. H. Snoeijer, Universality of Tip Singularity Formation in Freezing Water Drops, Phys. Rev. Lett. 113, 054301 (2014).

[25] P. F. Hao, C. J. Lv, and X. W. Zhang, Freezing of sessile water droplets on surfaces with various roughness and wettability, Appl. Phys. Lett. 104, 161609 (2014).

[26] F. Tavakoli, S. H. Davis, and H. P. Kavehpour, Spreading and arrest of a molten liquid on cold substrates, Langmuir 30, 10151 (2014).

[27] O. V. Voinov, Hydrodynamics of wetting, Fluid Dyn. 11, 714 (1976). 


\section{RIËLLE DE RUITER et al.}

[28] R. G. Cox, The dynamics of the spreading of liquids on a solid surface. Part 1. Viscous flow, J. Fluid Mech. 168, 169 (1986).

[29] T. Podgorski, J. M. Flesselles, and L. Limat, Corners, Cusps, and Pearls in Running Drops, Phys. Rev. Lett. 87, 036102 (2001).

[30] K. G. Winkels, I. R. Peters, F. Evangelista, M. Riepen, A. Daerr, L. Limat, and J. H. Snoeijer, Receding contact lines: From sliding drops to immersion lithography, Eur. Phys. J. Spec. Top. 192, 195 (2011).

[31] E. B. Giller and H. G. Drickamer, Viscosity of normal paraffins near the freezing point, Ind. Eng. Chem. 41, 2067 (1949).

[32] M. Amini and B. B. Laird, Kinetic Coefficient for Hard-Sphere Crystal Growth from the Melt, Phys. Rev. Lett. 97, 216102 (2006).

[33] A. I. Fedorchenko and A. B. Wang, Non-equilibrium solidification of the molten metal droplets impacting on a solid surface, Int. J. Heat Mass Transfer 50, 2463 (2007).

[34] D. M. Herlach, Dendrite growth kinetics in undercooled melts of intermetallic compounds, Crystals 5, 355 (2015).

[35] S. Schiaffino and A. A. Sonin, Molten droplet deposition and solidification at low Weber numbers, Phys. Fluids 9, 3172 (1997).

[36] M. Pasandideh-Fard, R. Bhola, S. Chandra, and J. Mostaghimi, Deposition of tin droplets on a steel plate: Simulations and experiments, Int. J. Heat Mass Transfer 41, 2929 (1998).

[37] S. D. Aziz and S. Chandra, Impact, recoil and splashing of molten metal droplets, Int. J. Heat Mass Transfer 43, 2841 (2000).

[38] R. Dhiman and S. Chandra, Freezing-induced splashing during impact of molten metal droplets with high Weber number, Int. J. Heat Mass Transfer 48, 5625 (2005).

[39] G. H. Rodway and J. D. Hunt, Thermoelectric investigation of solidification of lead I. Pure lead, J. Cryst. Growth 112, 554 (1991).

[40] F. Celestini and J. M. Debierre, Measuring kinetic coefficients by molecular dynamics simulation of zone melting, Phys. Rev. E 65, 041605 (2002).

[41] D. Buta, M. Asta, and J. J. Hoyt, Kinetic coefficient of steps at the Si(111) crystal-melt interface from molecular dynamics simulations, J. Chem. Phys. 127, 074703 (2007).

[42] J. Monk, Y. Yang, M. I. Mendelev, M. Asta, J. J. Hoyt, and D. Y. Sun, Determination of the crystal-melt interface kinetic coefficient from molecular dynamics simulations, Model. Simul. Mater. Sci. Eng. 18, 015004 (2010).

[43] J. J. Hoyt, M. Asta, and A. Karma, Atomistic simulation methods for computing the kinetic coefficient in solid-liquid systems, Interface Sci. 10, 181 (2002). 